ORIGINAL STUDY

\title{
Nasolacrimal duct obstruction: the relationship with nasal allergy
}

\author{
Desiderio Passali', Leandro Politi' ${ }^{1}$, Lei Chen ${ }^{2}$, Cemal Cingi ${ }^{3}$, Codrut Sarafoleanu ${ }^{4}$, \\ Pasquale Cassano 5
}

${ }^{1}$ Department of ENT, University of Siena, Siena, Italy

${ }^{2}$ Department of Otolaryngology Head and Neck Surgery, Chinese PLA General Hospital, Beijing, China

${ }^{3}$ Department of Otolaryngology Head and Neck Surgery, Osmangazi University, Faculty of Medicine, Eskisehir, Turkey

${ }^{4}$ Department of ENT, "Carol Davila” University of Medicine and Pharmacy, Bucharest, Romania

${ }^{5}$ Department of ENT, University of Foggia, Foggia, Italy

\section{ABSTRACT}

Nasolacrimal duct obstruction (NLDO) is a common event in clinical practice. The authors, all members of the Italian Society of Rhinology, analysed 100 cases from different ENT Departments. The causes of NLDO, according to the etiology, can be divided into two classes: idiopathic primary acquired nasolacrimal duct obstruction and secondary. The aim of the study was to describe an association between the sign and symptoms of chronic rhinosinusitis and a complaint of epiphora.

KEYWORDS: nasolacrimal duct obstruction, rhinologic diseases.

\section{INTRODUCTION}

Nasolacrimal duct obstruction (NLDO) is common in ophthalmologic clinics.

The causes of NLDO can be categorized, based on etiology, into two classes: idiopathic primary acquired nasolacrimal duct obstruction or secondary to various etiologies, including congenital, traumatic, inflammatory, neoplastic, and periocular radiotherapy and chemotherapy ${ }^{1}$. The condition is mostly diagnosed amongst middle-aged and older female patients ${ }^{2}$. The male/female ratio was $1: 2.8^{1,3}$, due to anatomically tighter and longer duct in women and the hormonal factor.

Increased incidence of NLDO in patients being treated for glaucoma with eye drops containing timolol is also to be reported. As a matter of fact, the drug induces conjunctival inflammation and fibrosis, fostering conditions to nasolacrimal duct stenosis ${ }^{4}$.

Both conjunctivitis and allergic rhinitis denote a similar pathogenetic mechanism; the contact with the allergen induces an inflammatory reaction which can promote NLDO.
The role of allergy in primary acquired NLDO was proved in small study groups, where rhinologic problems assume central importance in terms of etiol$\mathrm{ogy}^{4}$. Very important is also the role of concha bullosa, inferior turbinate hypertrophy, osteomeatal complex disease and maxillary sinusitis, in patients with primary unilateral acquired $\mathrm{NLDO}^{5}$. Allergic conjunctivitis is highly prevalent, and has close epidemiologic relationship with allergic rhinitis. Both conditions exhibit similar pathophysiologic mechanisms. Pathways of communication are assumed to increase the likelihood of an inflammatory reaction at both sites, following allergen exposure of nasal or ocular tissue ${ }^{1}$.

In case of allergies (rhinitis and conjunctivitis), the administration of topical nasal steroids assumes an important role in preventing NLDO. In fact, local application of these drugs proved effective in the reduction of both nasal symptoms and ophthalmologic manifestations. The effect can be explained by the suppression of the naso-ocular reflex, the down-regulation of inflammatory cells expression or the restoration of patency in the nasolacrimal duct ${ }^{6}$. 
The presence of positive bacteriological cultures is recurrent in NLDO (over $90 \%$ ). $59.4 \%$ of patients were affected by Gram-positive bacteria, with a prevalence of Staphylococcus epidermidis and Staphylococcus $\mathrm{Au}-$ reus. $37.5 \%$ were affected by Gram-negative bacteria, with a prevalence of Pseudomonas Aeruginosa. In a singular case Candida Albicans infection was identified, the condition in this specific case being most likely due to a prolonged use of ophthalmic antibiotics ${ }^{3}$.

It was suggested that nose and paranasal sinuses chronic inflammation might lead to epiphora because of inflammatory edema of the nasal end of the nasolacrimal duct ${ }^{7}$.

Nasal mucosa of patients undergoing dacryocystorhinoRX (dacryocystography) often shows histological evidence of chronic inflammation and fibrosis in respectively 32 and $50 \%$ of cases $^{8}$. In addition, it has been proved that $87 \%$ of patients with acquired nasolacrimal duct obstruction have nose and sinus abnormalities on computed tomography scanning, when compared with $63 \%$ of controls ${ }^{9}$.

This study aims to describe the association between the signs and symptoms of chronic rhinosinusitis and complaint of epiphora in a report study.

\section{MATERIAL AND METHODS}

In a multicentre study, patients with nasal obstruction and epiphora were recruited for this study. The group consisted of 100 adult patients, from different otorhinolaryngologic departments; patients who had undergone previous lacrimal surgery were excluded, so only 70 patients were selected.

All patients were studied with:

- Clinical history

- The subjective visual analogue scale (VAS) VAS was the average of five scores that each patient assigned to themselves and included values for five symptom parameters (nasal airways obstruction, rhinorrhea, headache, alteration of smell, sneezing). For every symptom parameter a patient could score ranging from 1 to 10 .

- Skin prick tests (Dermatophagoides mix, Grassmix allergy, Salicylates allergy and Compositae allergy)

- Examination of both nasal cavities with a flexible endoscope.

The most common symptoms reported by patients were episodic headaches and postnasal drip associated with epiphora.

Patients were also checked by an ophthalmologist, with extra attention being paid to lids and lacrimal puncta. In case of nasolacrimal duct obstruction diagnosis, a dacryocystorhinostomy was performed as a means of confirmation.
Before dacryocystorhinoRX, patients were all performed local anaesthesia (Figure 1).

Subsequently, contrast fluid was injected in the tear ducts (Figure 2), and the exam was performed (Figure 3, Figure 4).

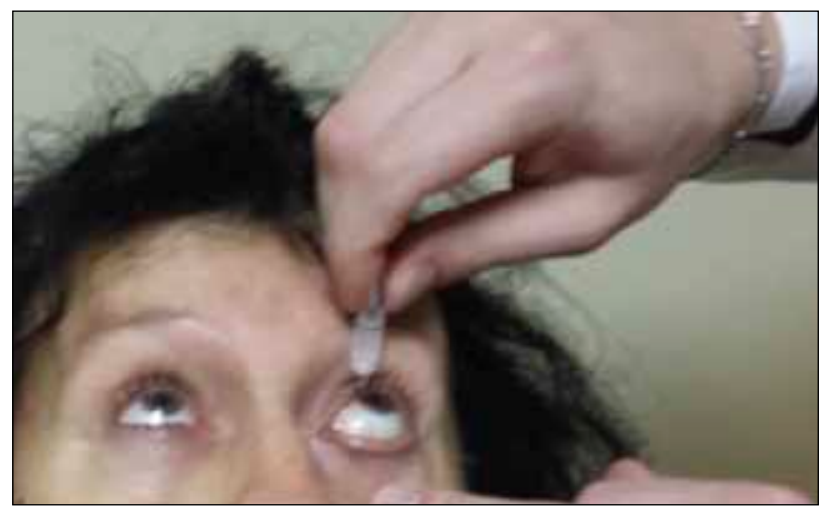

Figure 1 Local anaesthesia was performed before dacryocystorhinoRX.

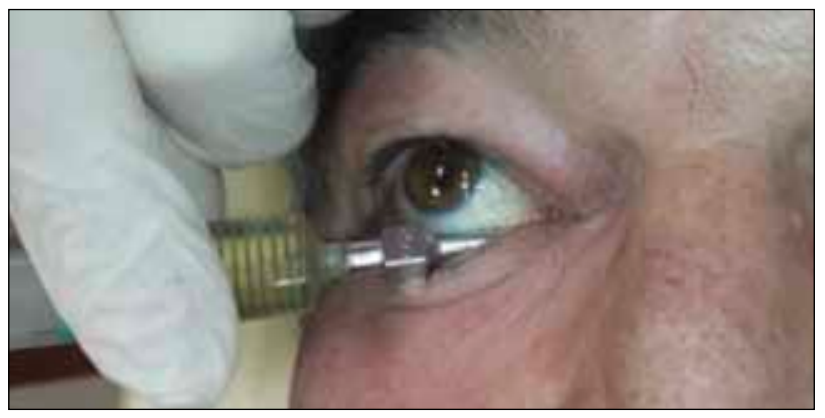

Figure 2 Contrast fluid was injected in the tear ducts.

\section{RESULTS}

We reported seventy patients with epiphora and nasal obstruction (50 female and 20 male). In fifty cases, dacryocystorhinoRX confirmed nasolacrimal duct obstruction and surgery was performed.

VAS value ranged from 4.4 to 7 . The results of nasal symptom scores are given in Graphic 1.

44 patients (35 with confirmed diagnosis of nasolacrimal duct obstruction and 9 in whom the anatomical obstruction was excluded by dacryocystorhinoRX) tested positive to Skin prick tests (21 to Dermatophagoides mix, 8 Grass-mix allergy and 6 to both) (Graphic 2).

The results of the endoscopic nasal examination are visible in Graphic 3. Each patient has undergone a full examination of both nasal cavities. We did not use any local anaesthetic or decongestant. The endoscopic evaluation revealed the presence of polyps, oedema and discharge. 12 patients were diagnosed with nasal polyp and HRTC (High-Resolution CT) was performed before surgery (FESS). 


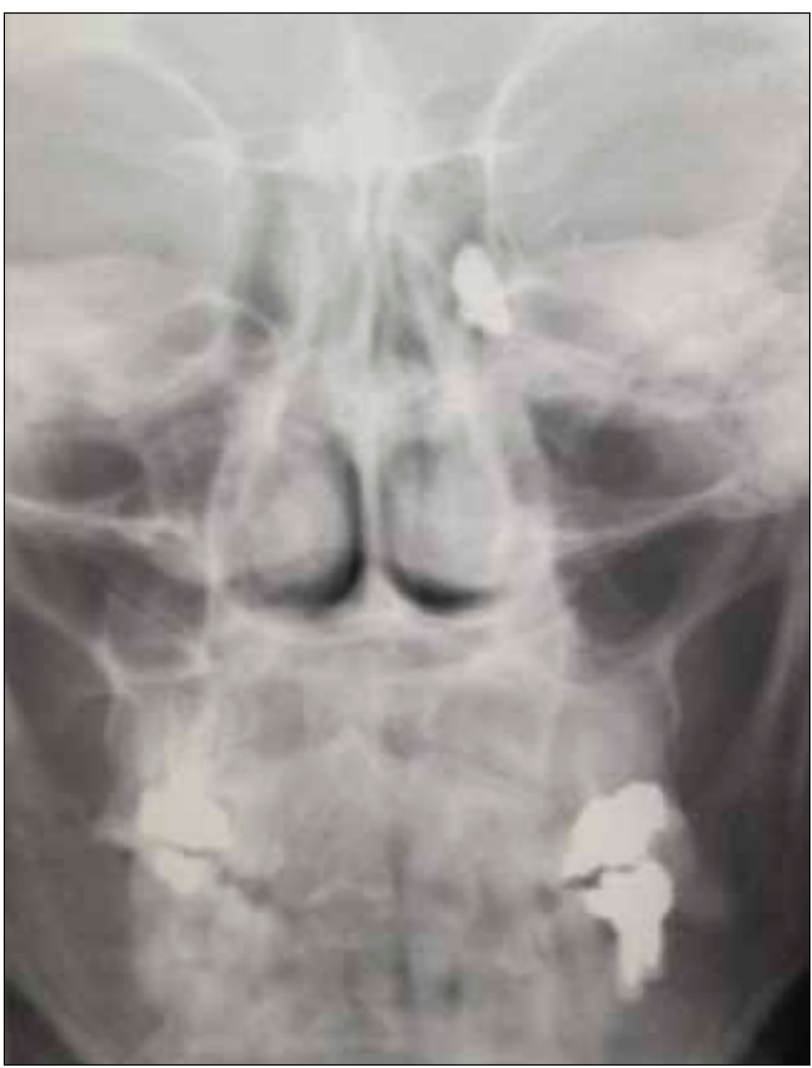

Figure 3 DacryocystorhinoRX result - frontal view - obstruction of the left lacrimal pathway.

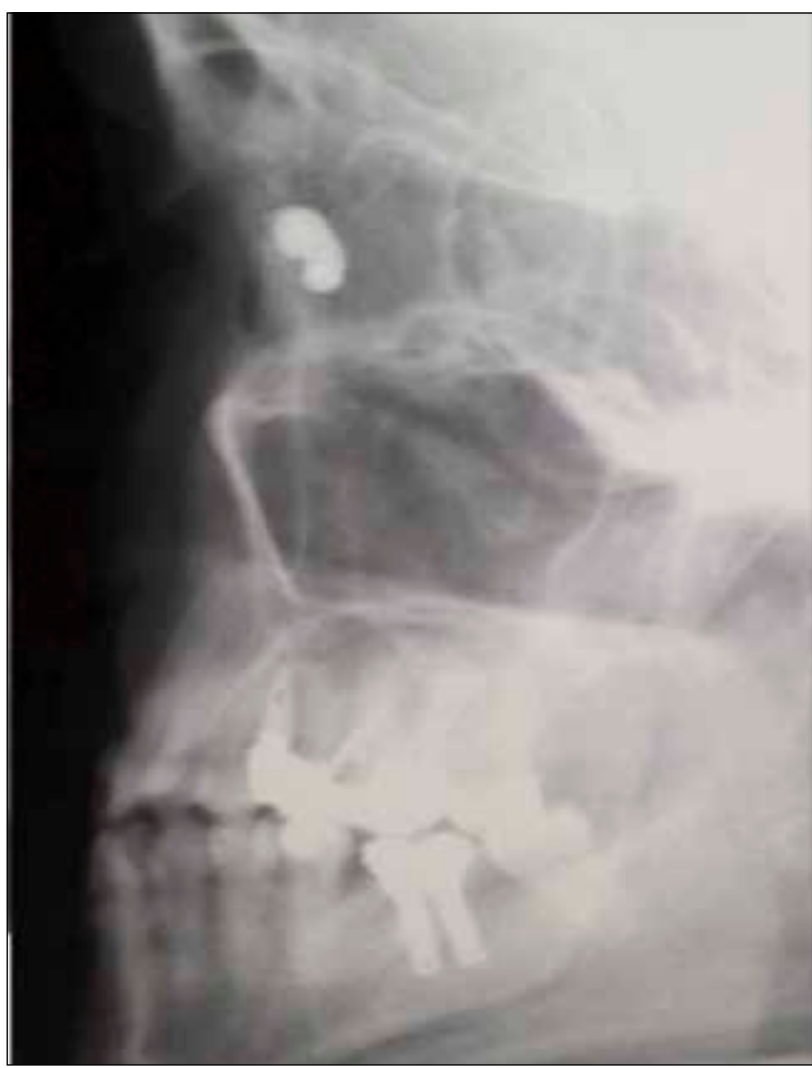

Figure 4 DacryocystorhinoRX result - lateral view - obstruction of the left lacrimal pathway.

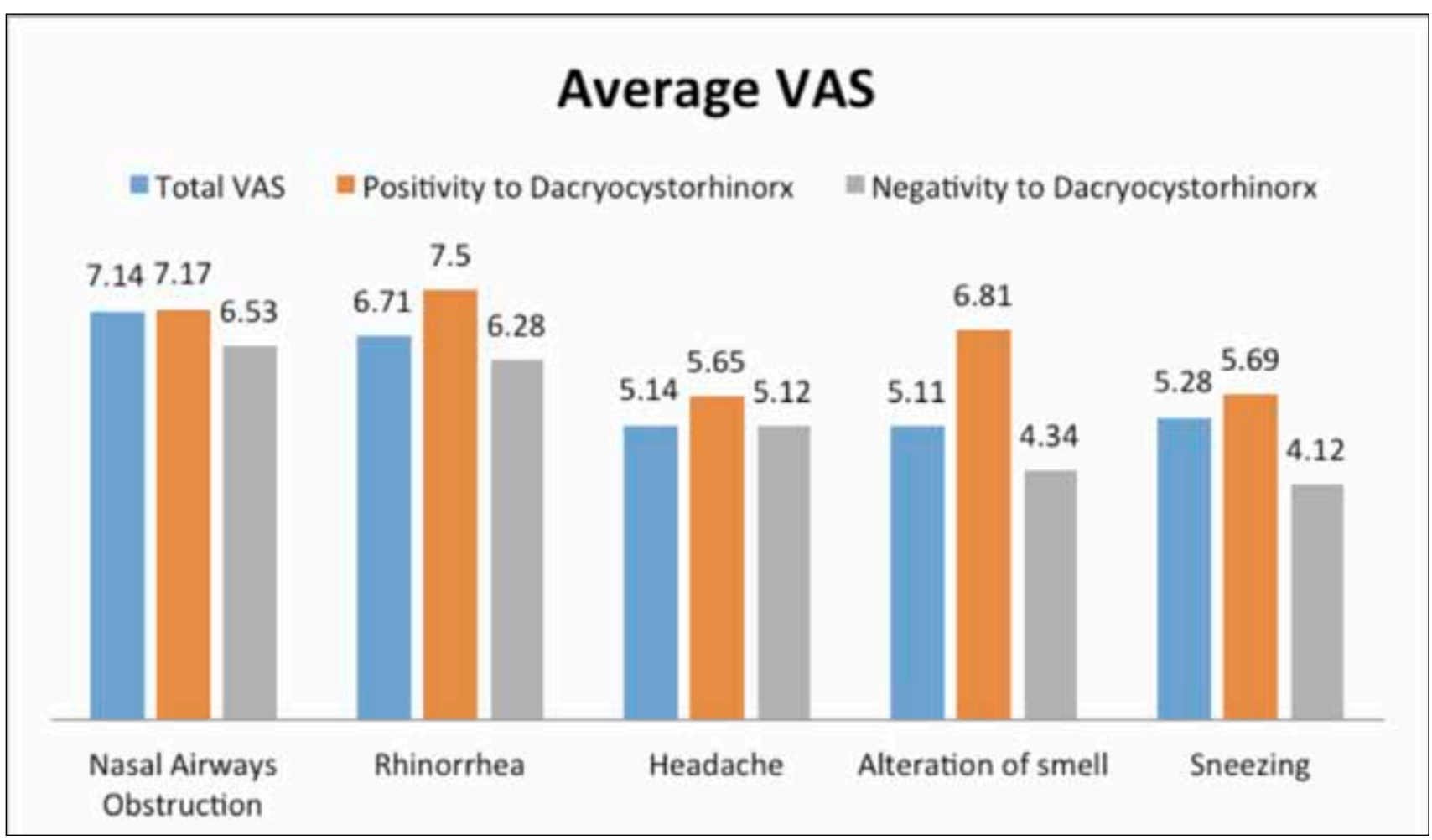

Graphic 1 VAS scores 


\section{Total Patients}

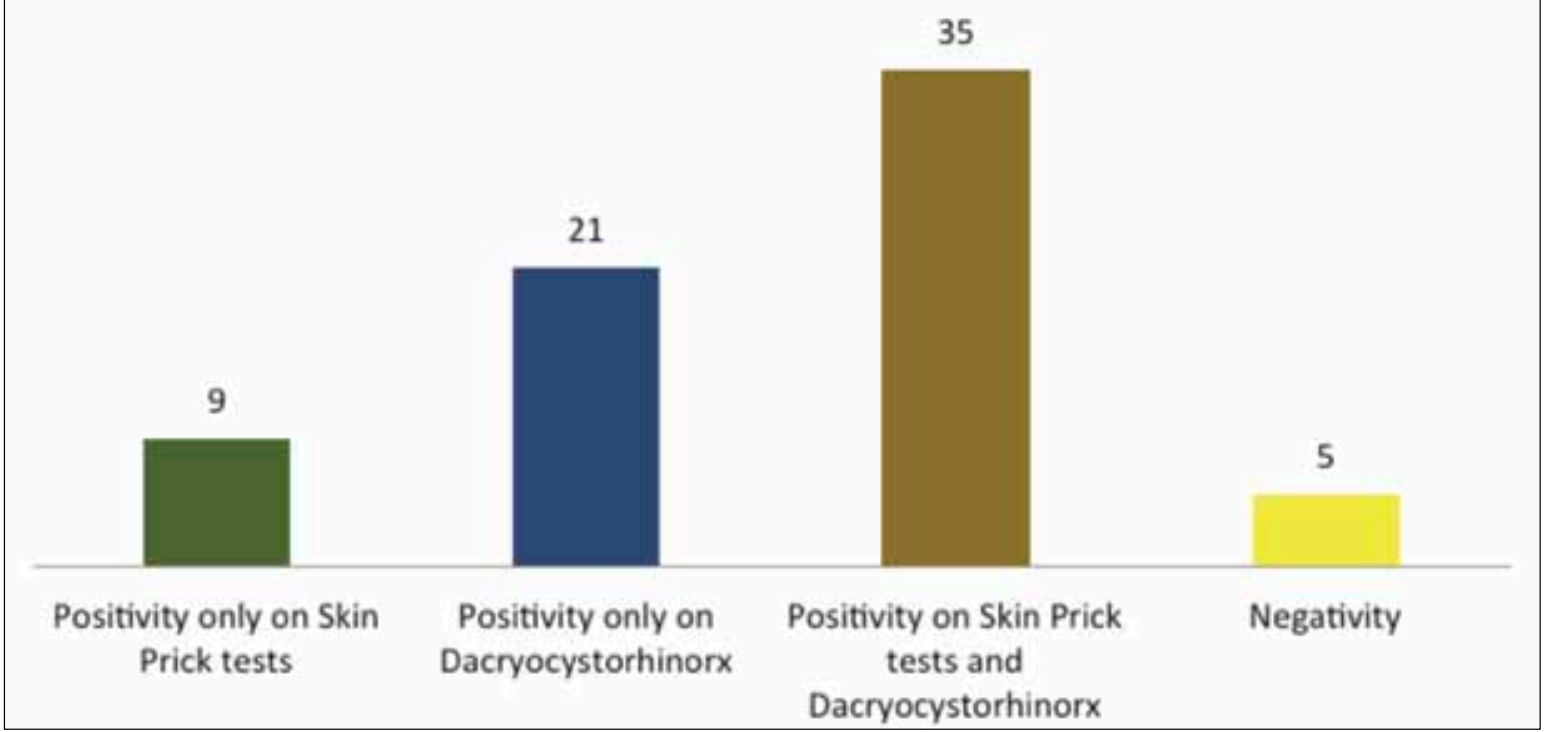

Graphic 2 The distribution of the patients according to dacryosytorhinoRX and skin prick tests results.

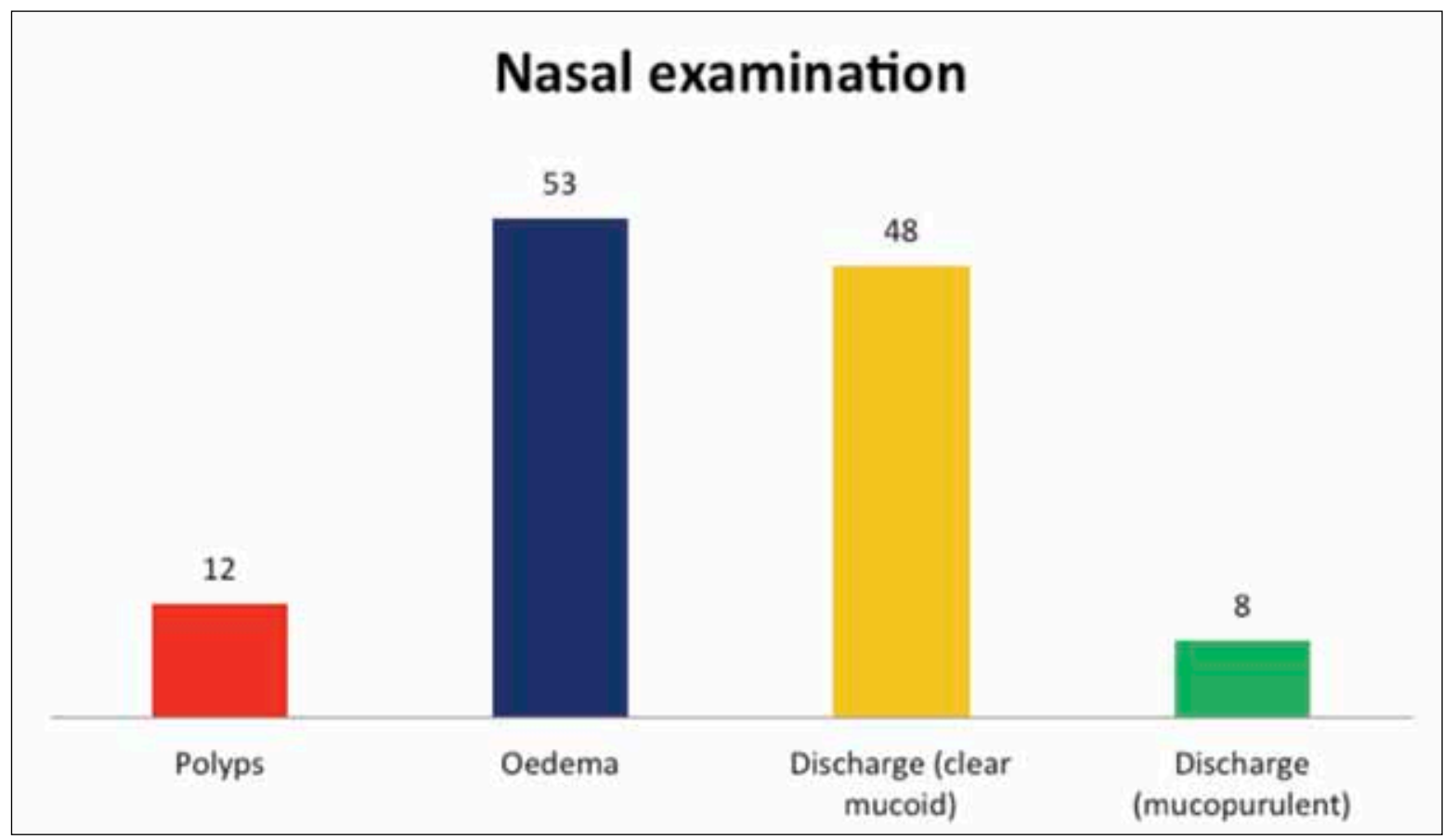

Graphic 3 Endoscopic nasal examination results

\section{DISCUSSIONS}

This study evidenced that patients diagnosed with nasal obstruction due to various etiologies (like allergic rhinosinusitis and other inflammatory etiologies) are often diagnosed with epiphora. The obstruction of the nasolacrimal duct had occurred in 50 out of 70 patients after radiographic control.

35 of 44 patients positive to Skin prick tests have
NLDO, so allergy seems to be related to this condition. Another nasal condition related to NLDO is oedema and discharge (clear mucoid) that can be observed with a flexible endoscope.

VAS was also higher in patient with positivity to dacryocystorhinoRX than the other controls (Graphic 1).

Allergic rhinitis and conjunctivitis exhibit similar pathophysiologic mechanisms. Pathways of communication are thought to increase the likelihood of an 
inflammatory reaction at both sites following allergen exposure of nasal or ocular tissue ${ }^{1}$. Intranasal therapies that initially demonstrated efficacy in nasal allergy symptoms also have been shown to alleviate symptoms of ocular allergy ${ }^{6}$ and can prevent epiphora if the underlying nasal condition can be identified and treated. In fact, edema of the nasal mucosa could lead to functional obstruction of the nasolacrimal duct orifice.

In our research, epiphora is often accompanied by nasal symptoms and sometimes precede in the latter years the onset of NLDO.

The early recognition of nasal symptoms of allergic and non-allergic patients is of primary importance to develop appropriate therapy and, therefore, prevent the development of epiphora.

The ENT evaluation should include a careful medical history focusing on the type of symptoms, their mode of onset and comorbidities.

Tests, such as allergy tests, like prick tests, and endoscopic examination, are needed for patient classification. To objectify the epiphora, ophthalmological evaluation and exams such dacryocystorhinoRX or dacryocystorhinoCT are necessary.

The presence of nasal polyps requires special attention. In fact, the subjects are not always allergic and the treatment of these forms becomes complex. Surgery is important in advanced stages, but relapse is a constant problem. Imperative is the need for adequate therapy post-operatively to reduce recurrence and complications such as NLDO. In these cases, X-ray examination, such as CT, allows not only to target the surgical program but also to evaluate the nasal lacrimal duct involvement.

It can be useful to treat conservatively the forms of epiphora of recent onset or linked to seasonal allergy. Surgery should be used where there is failure of medical treatment or in forms linked to anatomical anomalies.

Treatment of choice should be regarded to the use of topical corticosteroids, not only in the forms of allergic rhinosinusitis, and topical or oral antihistamines $^{6}$.
But to restore the patency of the nasolacrimal duct, surgery is required, especially in the forms of chronic rhinosinusitis when the duct fibrosis cannot be treated with medical therapy alone.

In conclusion, our results indicate that chronic rhinosinusitis, in particular allergic rhinosinusitis, are one of specific predisposing factors to the development of NLDO.

Conflict of interest: The authors have no conflict of interest.

Contribution of authors: All authors have equally contributed to this work.

\section{REFERENCES}

1. Nemet A.Y., Vinker S. - Associated morbidity of nasolacrimal duct obstruction - a large community based case-control study. Graefes Arch Clin Exp Ophthalmol., 2014;252(1):125-130. doi: 10.1007/s00417-0132484-3. Epub 2013 Oct 22.

2. McNabb A.A. - Lacrimal canalicular obstruction associated with topical ocular medication. Aust N Z J Ophthalmol., 1998;26(3):219-223.

3. Ali M.J., Ayyar A., Motukupally S.R., Sharma S., Naik M.N. - Bacteremia during dacryocystorhinostomy: results of intra-operative blood cultures. J Ophthalmic Inflamm Infect., 2014;4:27. doi: 10.1186/s12348-014-00277

4. Seider N., Miller B., Beiran I. - Topical glaucoma therapy as a risk factor for nasolacrimal duct obstruction. Am J Ophthalmol., 2008;145(1):120123. Epub 2007 Oct 29

5. Linberg J.V., McCormick S.A. - Primary acquired nasolacrimal duct obstruction. A clinicopathologic report and biopsy technique. Ophthamology, 1986;93(8):1055-1063.

6. Bielory L. - Allergic conjunctivitis and the impact of allergic rhinitis. Curr Allergy Asthma Rep., 2010;10(2):122-134. doi: 10.1007/s11882-0100087-1.

7. Garfin S.W. - Etiology of dacryocystitis and epiphora. Arch Ophthalmol. 1942;27(1):167-188. doi: 10.1001/archopht.1942.00880010185012.

8. Mauriello J.A. Jr, Palydowycz S., DeLuca J. - Clinicopathologic study of lacrimal sac and nasal mucosa in 44 patients with complete acquired nasolacrimal duct obstruction. Ophthal Plast Reconstr Surg., 1992;8(1):13-21.

9. Kallman J.E., Foster J.A., Wulc A.E., Yousem D.M., Kennedy D.W. Computed tomography in lacrimal outflow obstruction. Ophthalmology, 1997;104(4):676-682. 